\title{
UMA DESCRIÇÃO DAS ESCALAS DO CROSS-STAFF (1623) DE EDMUND GUNTER
}

\author{
A DESCRIPTION OF EDMUND GUNTER'S CROSS-STAFF SCALES (1623)
}

\author{
Andressa Gomes dos Santos ${ }^{1}$; Ana Carolina Costa Pereira ${ }^{2}$
}

\begin{abstract}
RESUMO
A Inglaterra, no século XVI e XVII, passou por grandes mudanças no que se refere às matemáticas por conta da necessidade de utilizá-las, por exemplo, na agrimensura, astronomia e navegação. Em decorrência dessa demanda, de direcionar as matemáticas às práticas, diversos tratados voltados para esse aspecto foram elaborados, muitos deles envolvendo a descrição e o uso de instrumentos, entre eles, pode-se destacar o tratado The description and vse of the Sector, the Crosse-staffe, and other instruments, for such as are studious of Mathematicall practise, de autoria de Edmund Gunter (1581 - 1626), datado de 1623, publicado em Londres, que aborda quatro instrumentos matemáticos: Setor, Cross-staff, Cross-bow e Quadrante. Visto isso, o estudo tem como objetivo apresentar o instrumento Cross-staff desenvolvido por Gunter (1623) e suas respectivas escalas. Esta pesquisa é um recorte de um estudo maior e se caracteriza como uma pesquisa documental, já que se apropria de um documento original para análise. Como resultado, foi apresentado um breve olhar sobre os séculos XVI e XVII, especialmente, em Londres, com foco em aspectos que estavam ligados ao contexto de produção do tratado The description and vse of the Sector, the Crosse-staffe, and other instruments..., no qual o instrumento está inserido, em seguida, são expostas as 12 escalas inscritas no Cross-staff de Gunter (1623). Percebe-se que esse instrumento possui escalas que incorporam diversos conhecimentos matemáticos, como trigonometria, unidades de medidas e proporção que, se explorados, estudados e direcionados ao ensino, podem contribuir com a construção e/ou ressignificação de conhecimentos.
\end{abstract}

Palavras-chave: Cross-staff; Edmund Gunter; História da matemática.

\begin{abstract}
England in the sixteenth and seventeenth century underwent major changes in terms of mathematics due to the need to use it, for example, in surveying, astronomy and navigation. As a result of this demand, to direct practices as mathematics, several treaties for this aspect were elaborated, many of them involving the description and use of instruments, among them the treatise The description and see of the Sector, the Crosse-staffe, and other instruments, for
\end{abstract}

\footnotetext{
${ }^{1}$ Licenciada em Matemática pela Universidade Estadual do Ceará (UECE). Estudante de Pós-Graduação no Instituto Federal de Educação, Ciência e Tecnologia do Ceará (IFCE). Bolsista da Fundação Cearense de Apoio ao Desenvolvimento Científico e Tecnológico (FUNCAP), Fortaleza, Ceará, Brasil. Endereço para correspondência: Av. Dr. Silas Munguba, 1700, Bloco das Coordenações - Sala 19, Itaperi, Fortaleza, Ceará, Brasil, CEP: 60.714.903. E-mail: andressa.gomes.santos06@ aluno.ifce.edu.br .

(iD) ORCID iD: https://orcid.org/0000-0003-1982-714X .

${ }^{2}$ Doutora em Educação pela Universidade Federal do Rio Grande do Norte (UFRN). Docente Adjunta na Universidade Estadual do Ceará (UECE), Fortaleza, Ceará, Brasil. Endereço para correspondência: Av. Dr. Silas Munguba, 1700, Bloco das Coordenações - Sala 19, Itaperi, Fortaleza, Ceará, Brasil, CEP: 60.714.903. E-mail: carolina.pereira@uece.br .

(iD) ORCID iD: https://orcid.org/0000-0002-3819-2381 .
} 
students of Mathematicall practice authored by Edmund Gunter (1581 - 1626) dated 1623 published in London that addresses four mathematical instruments, the Sector, Cross-staff, Crossbow and the Quadrant. In view of this, the study aims to present the Cross-staff instrument developed by Gunter (1623) and its different scales. This research is an excerpt from a larger study and is characterized as a documentary research, since it appropriates an original document for analysis. As a result, a brief look at the 16th and 17th century was presented, especially in London, focusing on aspects that were linked to the context of the production of the treaty The description and vision of the Sector, Crosse-staffe, and other instruments... in which the instrument is inserted, then the 12 scales registered in Gunter's Cross-staff (1623) are exposed. It is noticed that this instrument has scales that incorporate various mathematical knowledge such as trigonometry, units of measures and proportion that, if explored, studied and directed to teaching, can contribute to the construction and / or reframing of knowledge.

Keywords: Cross-staff; Edmund Gunter; History of mathematics. 


\section{Introdução}

A Europa, nos séculos XVI e XVII, estava passando por transformações quanto à forma de trânsito de conhecimentos. Nesse período, a universidade não era o local exclusivo para o estudo. Na Inglaterra, por exemplo, o conhecimento matemático estava presente em palestras realizadas além dos muros das universidades e nas lojas dos artesãos londrinos (ROSSI, 2001; CORMACK, 2017).

Com isso, em Londres, outros assuntos, que antes não eram abordados no Quadrivium $^{3}$, presente nas academias, como as práticas das matemáticas, passaram a ser estudados por causa, também, do interesse e do incentivo da coroa.

Assim, tratados matemáticos dos séculos XVI e XVII foram voltados para o estudo da matemática prática, para isso, diversas obras abordavam instrumentos que mobilizavam conhecimentos matemáticos, como os estudos de Digges (1573); Hood (1598) e Oughtred (1633).

Outro tratado que está direcionado para a matemática prática é o The description and yse of the Sector, the Crosse-staffe, and other instruments, for such as are studious of Mathematicall practise, de autoria de Edmund Gunter, publicado em Londres, datado de 1623, em sua primeira versão. Essa obra traz a descrição e o uso de quatro instrumentos, em particular: Setor, Cross-staff, Cross-bow e Quadrante.

Para este estudo, escolheu-se abordar o Cross-staff, um instrumento matemático que se destacava na prática de astronomia, agrimensura e navegação nos séculos XVI e XVII (CASTILlO, 2016). Desse modo, a pesquisa proposta tem como objetivo apresentar o instrumento Cross-staff desenvolvido por Gunter (1623) e suas respectivas escalas ${ }^{4}$.

Esta pesquisa é um recorte de um estudo mais amplo, para sua realização, foi feito um estudo documental da obra de Gunter (1623), especificamente, do segundo livro do tratado, que apresenta o instrumento foco deste artigo, caracterizando-o, assim, como uma pesquisa documental que "[...] é aquela em que os dados obtidos são estritamente

\footnotetext{
${ }^{3}$ O Trivium consiste no ensino de gramática, retórica e lógica, já o Quadrivium enquadra aritmética, geometria, música e astronomia (QUEIROZ, 1999).

${ }^{4}$ A escolha, tanto do tratado como do instrumento, também, está voltada para a construção de uma interface entre história e ensino, com a utilização de uma historiográfica atualizada proposta por Saito e Dias (2013); pelos preceitos apresentados em Silva (2018), para a seleção do documento histórico para ser inserido em sala de aula. Estudos, nessa perspectiva, podem ser encontrados em Alves e Pereira (2018); Santos e Pereira (2020a) e Santos e Pereira (2020b).
} 
provenientes de documentos, com o objetivo de extrair informações neles contidas [...]" (KRIPKA; SCHELLER; BONOTTO, 2015, p. 58).

Portanto, a próxima seção conta com um breve apanhado do contexto de elaboração do tratado de Edmund Gunter: The description and vse of the Sector, the Crosse-staffe, and other instruments..., no qual o Cross-staff está inserido.

\section{Breve contexto histórico do tratado The description and vse of the Sector, the Crosse- staffe, and other instruments...}

Diversos fatores, como a necessidade de se localizar em alto-mar e novos estudos sobre logaritmos, estavam presentes na sociedade londrina nos séculos XVI e XVII, que influenciaram e incentivaram a produção do The description and vse of the Sector, the Crosse-staffe, and other instruments... e de outros estudos que se concentraram no mesmo foco em meio às matemáticas da época, principalmente, no que se refere às práticas.

Nesse período, um centro foi criado por Thomas Gresham (1519 - 1579), para abordar correntes de estudos que ainda não estavam enraizadas nas universidades de Oxford e Cambridge. Assim, o Gresham College foi fundado, em 1597, com o intuito de reunir estudiosos de vários âmbitos, inclusive das matemáticas, em particular, voltadas para as práticas de navegação (HARTLEY; HINSHELWOOD, 1961).

A criação desse College decorre de incentivos da coroa inglesa, ainda no século XVI, no reinado de Elizabeth I, período em que já se valorizavam estudos em práticas que pudessem contribuir, especialmente, com a navegação, visto a necessidade de se localizar em alto-mar (ASH, 2004). Mediante isso,

[...] os elisabetanos apoiavam a ciência prática e ofereciam vários incentivos atraentes aos matemáticos ingleses para se dedicarem a ela. Por um lado, a sociedade elisabetana ofereceu-lhes a oportunidade de ganhar a vida com matemática aplicada, por meio de patrocínio, emprego em uma empresa comercial no exterior ou no comércio de livros. Além disso, como certas áreas da matemática prática - acima de tudo, matemática da navegação - estavam ligadas à política e ao bem-estar da nação, os elisabetanos incentivaram o serviço nessas áreas, tornando-o um dever patriótico (ROSS, 1975, p. 49, tradução nossa) ${ }^{5}$.

5 “ [...] the Elizabethans were supporters of practical science, and offered a number of attractive inducements to English mathematicians to devote themselves to it. For one thing, Elizabethan society offered them the opportunity to earn their living in applied mathematics, through patronage, employment with an overseas trading company, or in the book trade. Also, because certain areas of practical mathematics - above all, 
No que diz respeito aos assuntos palestrados no Gresham College, estavam presentes os assuntos de divindade, direito civil, física, retórica, astronomia, geometria e música (CHARTRES; VERMONT, 1998). Essa instituição buscava abordar temáticas que não eram muito discutidas nas universidades, logo, tratando, por exemplo, a geometria e a astronomia de forma prática.

O Gresham College destacava dois assuntos do Quadrivium, a disciplina de geometria foi ministrada por Henry Briggs (1561 - 1630) até o ano de 1920, em decorrência de sua amizade com Edmund Gunter, Briggs o indicou ao cargo de professor de astronomia, depois de Thomas Williams ${ }^{6}$ ter renunciado seu posto em 1619.

Edmund Gunter ${ }^{7}$ nasceu em Hertfordshire, na Inglaterra, em 1581, foi aluno da Westminster School e da Christ Church em Oxford, obteve o título de bacharel em Artes em 1603 e de Mestre em Artes três anos depois (WARD, 1740; COTTER, 1981). Seu ingresso no Gresham College permitiu uma aproximação com Briggs, que estava se aprofundando em estudos sobre logaritmos de base decimal ${ }^{8}$. Um fruto dessa colaboração foi o tratado Canon Triangvlorvm, de Gunter, publicado em 1620, em que o autor apresenta várias tabelas de senos e tangentes relacionadas com os logaritmos desenvolvidos por Briggs.

Esse estudo de 1920, de Gunter, é importante para construção de algumas escalas do instrumento Cross-staff, apresentado no seu tratado The description and vse of the Sector, the Crosse-staffe, and other instruments, for such as are studious of Mathematicall practise (Figura 1), publicado, em sua primeira versão, em 1623, impresso por William Jones e vendido por Edmund Weaver em Londres.

navigational mathematics - were tied to the policy and well-being of the nation, the Elizabethans additionally encouraged service in these areas by making it a patriotic duty" (ROSS, 1975, p. 49).

${ }^{6}$ Pouco se sabe sobre esse estudioso. De acordo com Ward (1740), Thomas Williams estava ocupando um cargo de professor de astronomia no Gresham College, em meados de 1613 e escreveu uma nota renunciando seu posto na instituição em 1619.

${ }^{7}$ Edmund Gunter escreveu um manuscrito sobre o instrumento Setor e publicou cinco tratados, a saber: New projection of the Sphere, Canon triangvlorvm, De Sectore \& Radio, The description and vse of the Sector, the Crosse-Staffe, and other instruments... e The description and use of his majestie's dials in Whitehall Garden.

${ }^{8}$ Henry Briggs estudou, juntamente com John Napier (1550 - 1617), os logaritmos de base decimal. Vale ressaltar que Napier desenvolveu dois tratados sobre esse assunto, entretanto, seus logaritmos não eram desenvolvidos em base decimal (HAVIL, 2014). 
Figura 1 - The description and vse of the Sector, the Crosse-staffe, and other instruments...

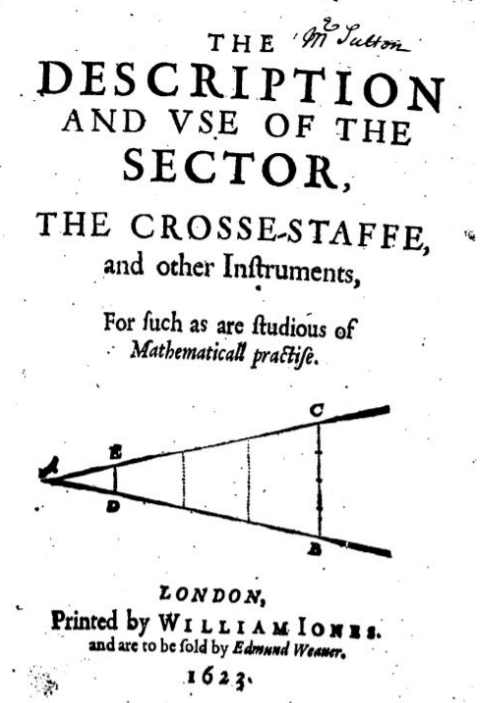

Fonte: Gunter (1623, contracapa).

Esse tratado de Gunter (1623) é dividido em dois livros. O primeiro traz a descrição e o uso do instrumento Setor, o segundo livro trata sobre o Cross-staff (Figura 2) e outros dois instrumentos, a saber: o Cross-bow e o Quadrante, dispostos em dois apêndices respectivamente.

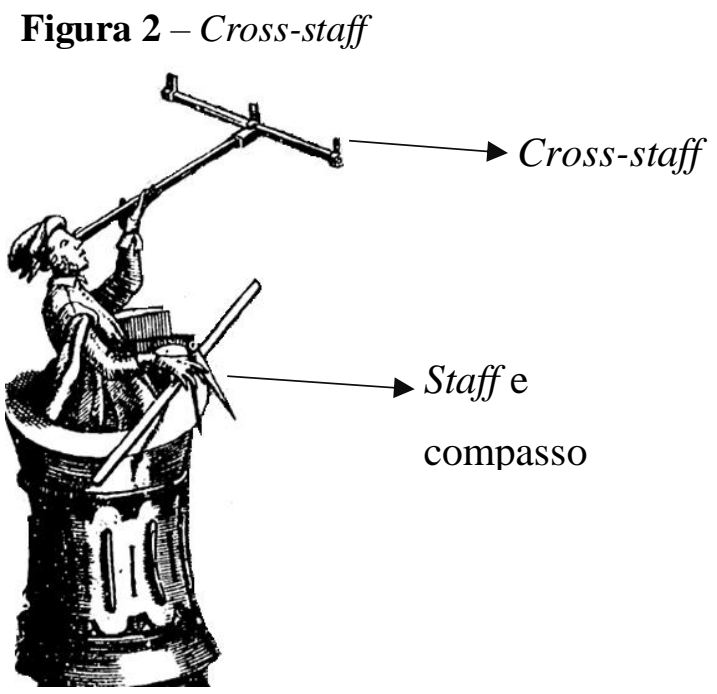

Fonte: Adaptado de Gunter (1623, frontispício).

$\mathrm{Na}$ próxima seção, são abordadas as escalas que compõem o Cross-staff, separadas pelas partes do instrumento, já que ele conta com cinco, em particular, o cross com três miras e o staff. Assim, apresentam-se as escalas inscritas no cross e no staff. 


\section{As escalas do instrumento Cross-staff, de Edmund Gunter}

O livro que trata sobre o Cross-staff começa com uma definição do instrumento e indica quem comumente o manuseia, o autor destaca que

O Cross-Staff 9 é um instrumento muito conhecido por homens do mar, e muito usado por astrônomos antigos e outros, servindo astronomicamente para observação de altitude e ângulos de distância no céu, geometricamente para alturas e distâncias perpendiculares na terra e no mar (GUNTER, 1623, p. 1, tradução nossa) ${ }^{10}$.

O autor deixa claras as dimensões do Cross-staff (Figura 3) instrumento, sendo o staff com medida igual a uma jarda completa, que é igual a 36 polegadas e o cross medindo 26 polegadas e $\frac{1}{5}$. Em outras palavras, pode-se obter o comprimento dessas partes pela proporção de 360 e 262 (GUNTER, 1623).

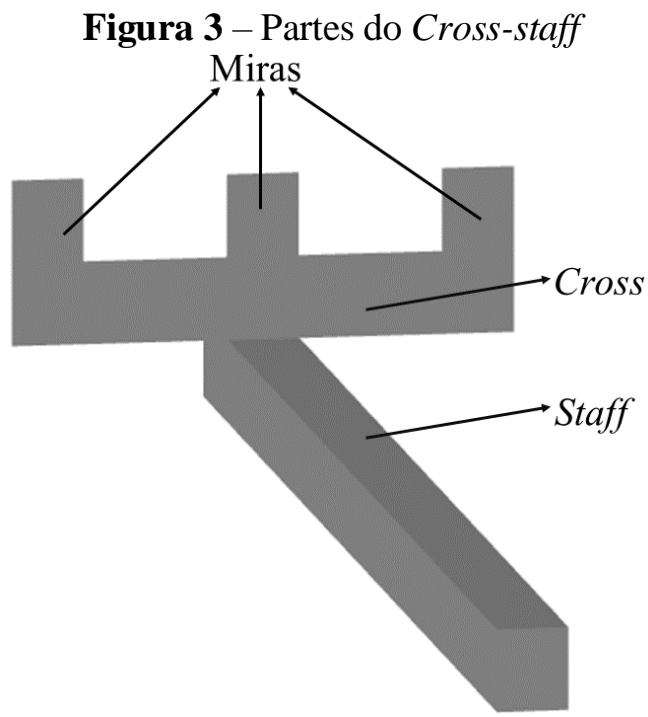

Fonte: Elaborada pela autora (2021).

Esse Cross-staff se diferenciava dos demais da época, pois, segundo Roche (1981), Edmund Gunter elaborou escalas inéditas para esse instrumento, especificamente, para o staff, o que é evidenciado pelo próprio autor quando ele relata que

A descrição e vários usos do mesmo [Cross-staff] são impressos, por Gemma Frisius em latim, em inglês por Dr. Hood. Eu diferi algo dos dois, na proposta deste Staff, mas assim como suas regras podem ser aplicadas a ele, e todas as

\footnotetext{
${ }^{9}$ No documento original, Gunter (1623) traz a denominação Crosse-staffe, porém optou-se por utilizar o termo em inglês moderno: Cross-staff, para se referir ao instrumento.

${ }^{10}$ Lê-se em inglês: "The Crosse-Staffe is an instrument well knowe to our Sea-men, and much used by the ancient astronomers and others, serving astronomically for observation of altitude and angles of distance in the heavens, Geometrically for perpendicular Heights and distances on land and sea" (GUNTER, 1623, p. 1).
} 
suas proposições são elaboradas por ele: e, portanto, referindo o leitor a seus livros, serei mais breve na explicação daquilo que pode ser aplicado deles aos meus e, portanto, chegamos ao uso daquelas linhas que são da minha adição, não existentes até agora (GUNTER, 1623, p. 1, tradução nossa) ${ }^{11}$.

Confirma-se, então, que Gunter (1623) adicionou escalas no staff desse instrumento, que foram desenvolvidas por ele. Assim, foram apresentados aspectos gerais desse instrumento, sabendo que há escalas inscritas no cross e no staff desse artefato, observam-se, nas próximas seções, as escalas dispostas em cada um.

\section{As escalas do staff}

Conforme Gunter (1623, p. 2, tradução nossa) ${ }^{12}$, o staff conta com sete escalas, divididas em quatro tipos: "uma delas serve para medir e prolongar: uma para observação de ângulos: uma para o mapa do mar; e as quatro outras, para trabalharem proporções de vários tipos". Observa-se essa classificação na Figura 4.

${ }^{11}$ Lê-se em inglês: "The description and severall use of it are extant in print, by Gemma Frisius in Latin, in English by Dr. Hood. I differ something from them both, in the projection pf this Staffe, but so, as their rules may be applied unto it, and all their propositions be wrought by it: and therefore referring the reader to their bookes, I shal be briefe in the explanation of that which may be applied from theirs unto mine, and so come to the use of those lines which are of my addition, not extant heretofore" (GUNTER, 1623a, p. 1). ${ }^{12}$ Lê-se no original: "One of them serves for measure and protraction: one for observation of angles: one for the Sea-chart; and the foure others for working of proportions in severall kinds" (GUNTER, 1623, p. 2). 


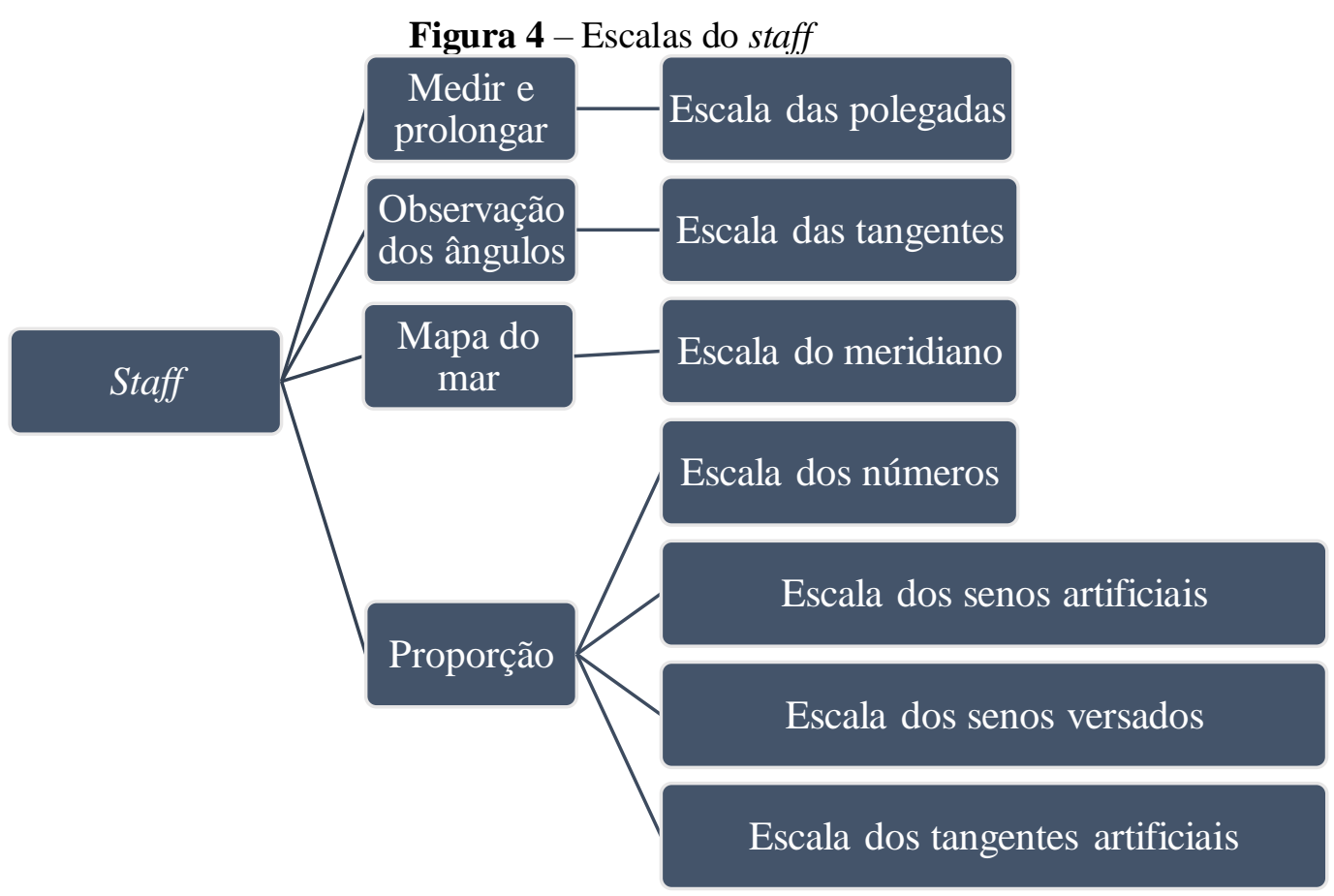

Fonte: Elaborada pela autora (2021).

Gunter $(1623 \text {, p. 2, tradução nossa })^{13}$, para a escala das polegadas, descreve que "a jarda completa foi dividida igualmente em 36 polegadas, e cada polegada subdividida, primeiro em dez partes e depois cada décima parte na metade". Para a construção, devese considerar o comprimento da jarda do staff e dividi-la em 36 polegadas, em seguida, pela décima parte e essa última dividir pela metade.

Já a escala das tangentes do staff é conhecida pelos números duplos definidos em ambos os lados, começando de um lado em 20 e terminando em 90 e, do outro lado, em 40 e terminando em 180, de acordo com os graus de um quadrante (GUNTER, 1623). O autor não dá mais detalhes sobre essa escala, como dito por ele, anteriormente, que não daria ênfase no que já era usado em outros instrumentos da época.

A próxima escala do staff a ser descrita é a do "[...] meridiano do mapa do mar, de acordo com a projeção de Mercators do equinocial a 58gr. de latitude e pode ser conhecido pela letra $M$ e pelos números 1.2. 3. 4. até 58” (GUNTER, 1623, p. 2, tradução nossa) ${ }^{14}$.

\footnotetext{
${ }^{13}$ Lê-se no original: "The whole yard being divided equally into 36 inches, and each inch subdivided, first into ten parts, and then each tenth part into halfes" (GUNTER, 1623, p. 2).

${ }^{14}$ Lê-se no original: "[...] meridian of a Sea-chart, according to Mercators projection from the equinoctiall to 58gr. of latitude, and may be knowne by the letter M, and the numbers 1. 2. 3. 4. unto 58." (GUNTER, 1623, p. 2).
} 
As escalas das proporções são quatro: a dos números, das tangentes artificiais, dos senos artificiais e dos senos versados, e todas têm divisões desiguais. Elas foram desenvolvidas pelo autor e são inéditas até então. Gunter (1623, p. 2, tradução nossa) ${ }^{15}$ descreve que

\begin{abstract}
A escala dos números anotada com a letra $\mathrm{N}$ é dividida desigualmente em 1000 partes e numerada com 1.2.3.4. até 10 .

A escala das tangentes artificiais é anotada com a letra $\mathrm{T}$, dividida desigualmente em 45 graus, e numerada nos dois sentidos, para o tangente e o seu complemento.

A escala dos senos artificiais, anotada com a letra $S$, dividida desigualmente em 90 graus e numerada com 1.2.3. 4. até 90 graus.

A escala dos senos versados para maior facilidade em encontrar a hora e o azimute $^{16}$, notada com V, dividiu-se desigualmente em cerca de $164 \mathrm{gr}$. $50 \mathrm{~m}$. numerada em sentido contrário com 10. 20. 30. até 164.
\end{abstract}

Gunter (1623) indica, para a construção da escala dos números, a utilização do tratado Logarithmorum Chilias Prima, de Henry Briggs e, para as demais escalas das proporções, ele cita o uso do seu tratado Canon Triangvlorvm.

Essas sete escalas são inscritas no staff, percebem-se vários elementos matemáticos em cada uma dessas escalas, que podem ser potencialmente didáticas. Algumas são usadas com o auxílio do compasso, como as escalas das proporções e as demais são utilizadas em conjunto com escalas dispostas no cross.

\title{
Escalas inscritas no cross
}

No cross, são inscritas mais cinco escalas, porém elas não são classificadas como as do staff: A saber, a escala da tangente de 20, da tangente de 30, das polegadas, das cordas e a continuação da escala do meridiano (Figura 5).

\footnotetext{
${ }^{15}$ Lê-se em inglês: "The line of numbers noted with the letter $\mathrm{N}$, divided unequally into 1000 parts, and numbred with 1.2.3.4. unto 10 .

The line of artificial tangents is noted with the letter T; divided unequally into 45 degrees, and numbred both ways, for the Tangent and the complement.

The line of artificial sines, noted with the letter S; divided unequally into 90 degrees, and numbred with 1 . 2.3. 4. unto 90 .

The line of versed sines for more ease finding the houre and azimuth, noted with $\mathrm{V}$, divided unequally into about 164gr. 50m. numbred backward with 10. 20. 30. unto 164” (GUNTER, 1623, p. 2).

${ }^{16}$ Distância angular medida sobre o horizonte.
} 


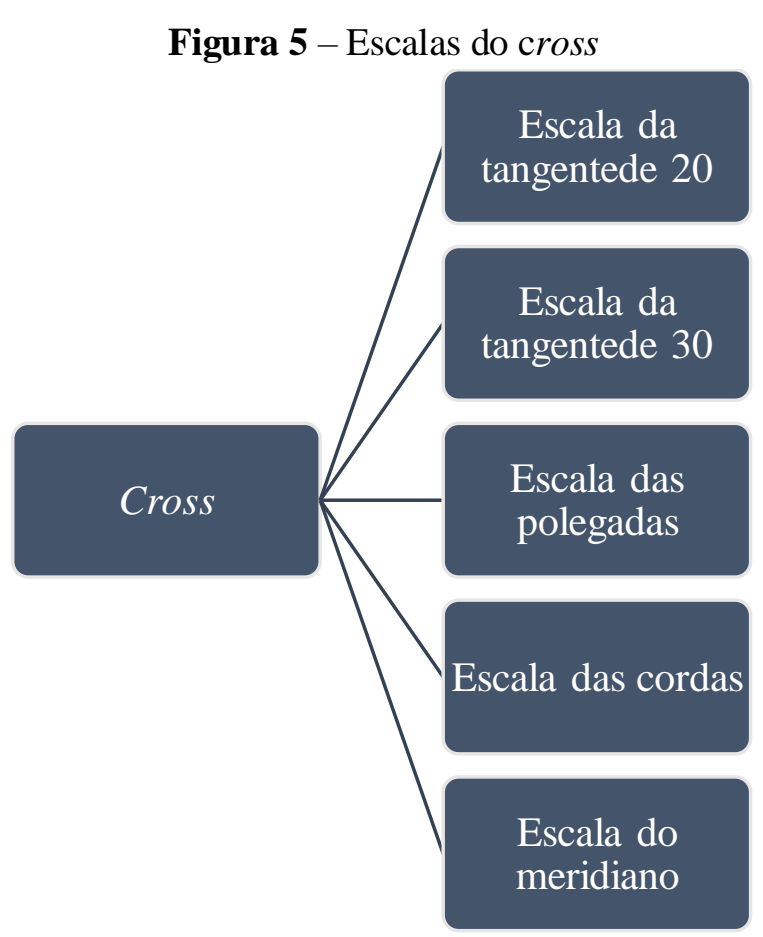

Fonte: Elaborada pela autora (2021).

A primeira descrita é a escala da tangente de 20, Gunter (1623, p. 3, tradução nossa) ${ }^{17}$ descreve a "escala de Tangente de $36 \mathrm{gr}$. $3 \mathrm{~m}$. numerada por 5 . 10. 15. até 25: o meio é de $20 \mathrm{gr}$; e, portanto, eu chamo de tangente de 20; e isso corresponde até $20 \mathrm{gr}$. $\mathrm{Na}$ tangente no Staff".

Em seguida, o autor destaca mais "uma escala de Tangente de $49 \mathrm{gr}$. $6 \mathrm{~m}$. numerada por 5. 10.15. até 45; o meio é de $30 \mathrm{gr}$. e tem respeito até $30 \mathrm{gr}$. na tangente de Staff, pelo que eu chamo de tangente de 30" (GUNTER, 1623, p. 3, tradução nossa) ${ }^{18}$.

Essas escalas de tangente de 20 e de 30 são utilizadas com a escala das tangentes inscrita no staff. A próxima escala mencionada por Gunter (1623, p. 3, tradução nossa) ${ }^{19}$ é "uma escala de polegadas numerada com 1. 2. 3. até 26; cada polegada subdividiu-se igualmente em dez partes, respondendo à escala de polegadas sobre a Staff'. Essa escala é manuseada juntamente com a escala das polegadas disposta no staff.

${ }^{17}$ Lê-se no original: "A tangent line of $36 \mathrm{gr} .3 \mathrm{~m}$. numbred by 5. 10. 15. unto 25: the midst whereof is at $20 \mathrm{gr}$; and therefore I call it the tangent of 20; and this hath respect unto $20 \mathrm{gr}$. In the tangent on the Staffe" (GUNTER, 1623, p. 3).

${ }^{18}$ Lê-se no original: "A Tangent line of $49 \mathrm{gr}$. $6 \mathrm{~m}$. numbred by 5. 10.15. unto 45 ; the midst whereof is at $30 \mathrm{gr}$. and hath respect unto $30 \mathrm{gr}$. in the tangent on the Staffe, whereupon i call it the tangent of 30 " (GUNTER, 1623, p. 3).

${ }^{19}$ Lê-se no original: "A line of inches numbred with 1.2.3. unto 26; each inch equally subdivided into ten parts, answerable to the inch line upon the Staffe" (GUNTER, 1623, p. 3). 
Em seguida, Gunter (1623, p. 3, tradução nossa $)^{20}$ descreve

uma escala das cordas diversas, correspondente a um círculo de doze polegadas de semidiâmetro, numerado com 10. 20. 30. até 60: outro a um semidiâmetro de um círculo de polegadas fixas; e o terceiro a um semidiâmetro de um círculo de três polegadas; ambos numerados com 10. 20. 30. a 90.

Essa escala é utilizada independente das demais, é necessário o auxílio de um compasso para manuseá-la. Por fim, Gunter (1623, p. 3, tradução nossa $)^{21}$ apresenta "uma continuação da escala do meridiano de $57 \mathrm{gr}$. De latitude até $76 \mathrm{gr}$; e a partir de $76 \mathrm{gr}$. Até 84gr”. Essa escala é uma continuação da escala do meridiano inscrita no staff.

Cada uma dessas escalas, tanto as dispostas no staff como no cross, tem uma finalidade e todas mobilizam algum aspecto matemático, seja nas suas construções ou em seus manuseios, assim, o intuito foi apresentar um possível recurso a ser implementado em sala de aula mediante estudo sobre suas aplicações.

\section{Considerações finais}

A Inglaterra passou por mudanças no que diz respeito ao ensino das matemáticas nos séculos XVI e XVII. Nesse período, a prática matemática estava em alta pela demanda de estudos sobre esse aspecto e pela necessidade de instrumentos que mobilizavam esse conhecimento.

Um instrumento bastante utilizado nesse período, que envolve conhecimentos matemáticos, é o Cross-staff. Neste estudo, destacou-se o desenvolvido por Edmund Gunter e apresentado em seu tratado: The description and vse of the Sector, the Crossestaffe, and other instruments..., publicado em 1623.

Portanto, apresentou-se um breve contexto de Londres nos séculos XVI e XVII, âmbito no qual o tratado que contém o Cross-staff, de Gunter (1623), foi elaborado e foram expostas as escalas inscritas nas partes desse instrumento, respectivamente, no staff e no cross. Logo, alcançando o objetivo deste artigo de apresentar essas escalas.

Desse modo, conhecendo o instrumento Cross-staff, percebe-se que ele pode ser um recurso para ser aliado ao ensino de matemática, já que mobiliza conhecimentos, de

\footnotetext{
${ }^{20}$ Lê-se no original: "A line of severall chords, one answerable to a circle of twelve inches semidiameter, numbred with 10. 20.30. Unto 60: another to a semidiameter of a circle of fix inches; and the third to a semidiameter of a circle of three inches; both numbred with 10. 20. 30. unto 90" (GUNTER, 1623, p. 3). ${ }^{21}$ Lê-se no original: "A continuation of the meridian line from $57 \mathrm{gr}$. Of latitude unto 76gr; and from $76 \mathrm{gr}$. To 84gr” (GUNTER, 1623, p. 3).
} 
acordo com a matemática do século XXI, sobre trigonometria, unidades de medida e proporção.

Assim, é possível que o professor de matemática identifique e reconheça na história outras maneiras de incorporá-la ao ensino, podendo levar à sala de aula formas de reconfigurar um conhecimento a partir disso.

\section{Referências}

ALVES, Verusca Batista; PEREIRA, Ana Carolina Costa. O instrumento "círculos de proporção" exposto na obra de William Oughtred (1633): um elemento na interface entre história e ensino de matemática. Revista de Produção Discente em Educação Matemática, São Paulo, v. 7, n. 2, p. 89-108, 11 set. 2018. Disponível em: http://revistas.pucsp.br/pdemat/article/view/39043. Acesso em: 11 maio 2020.

ASH, Eric H.. Power, Knowledge, and expertise in Elizabethan England. [S.I.]: The Johns Hopkins University Press, 2004.

CASTILLO, Ana Rebeca Miranda. Um estudo sobre os conhecimentos matemáticos incorporados e mobilizados na construção e uso do báculo (cross-staff) em A Boke Named Tectonicon de Leonardo Digges. 2016. 120 f. Tese (Doutorado) - Curso de Educação Matemática, Pontifícia Universidade Católica de São Paulo, São Paulo, 2016.

CHARTRES, Richard; VERMONT, David. A brief history of Grasham College 15971997. [S.I.]: Gresham College, 1998.

CORMACK, Lesley B.. Mathematics for Sale: Mathematical Practitioners, Instrument Makers, and Communities of Scholars in Sixteenth-Century London. In: CORMACK, Lesley B.; WALTON, Steven A.; SCHUSTER, John A. (ed.). Mathematical Practitioners and the Transformation of Natural Knowledge in Early Modern Europe. 45. ed. Cham: Springer, 2017. (Studies in History and Philosophy of Science).

COTTER, Charles H.. Edmund Gunter (1581-1626). Journal Of Navigation, [S.L.], v. 34, n. 3, p. 363-367, set. 1981. Cambridge University Press (CUP). http://dx.doi.org/10.1017/s0373463300047998. Disponível em: https://www.cambridge.org/core/journals/journal-of-navigation/article/edmund-gunter15811626/842FBA9BED16ABDA5B562A793C8036E7. Acesso em: 09 set. 2020.

DIGGES, Thomas. Alae Seu Scalae Mathematicae. London: Anno Domini, 1573.

HARTLEY, Harold; HINSHELWOOD, Cyril. Gresham College and the Royal Society. Notes And Records Of The Royal Society Of London, [S.L.], v. 16, n. 1, p. 125-135, 30 abr. 1961. The Royal Society. http://dx.doi.org/10.1098/rsnr.1961.0031. Disponível em: https://royalsocietypublishing.org/doi/10.1098/rsnr.1978.0001. Acesso em: 09 set. 2020. 
HAVIL, Julian. John Napier: life, logarithms, and legacy. Princeton: Princeton University Press, 2014.

GUNTER, Edmund. Canon triangvlorvm. London: Gulielmus Jones, 1620.

GUNTER, Edmund. The Description and use of the sector. The Crosse-staffe and other instruments, For such as are studious of Mathematicall practise. London: William Jones, 1623.

GUNTER, Edmund. The Description and use of the sector. The Crosse-staffe and other instruments. For such as are studious of Mathematicall practise. London: William Jones, 1624.

HOOD, Thomas. The making and vse of the Geometricall Instrument, called a Sector. London: John Windet, 1598.

KRIPKA, Rosana Maria Luvezute; SCHELLER, Morgana; BONOTTO, Danusa de Lara. La investigación documental sobre la investigación cualitativa: conceptos y caracterización.. Revista de Investigaciones Unad, [s.1.], v. 14, n. 2, p. 55, 24 nov. 2015. Universidad Nacional Abierta y a Distancia. http://dx.doi.org/10.22490/25391887.1455. Disponível em: https://doi.org/10.22490/25391887.1455. Acesso em: 11 jun. 2020.

OUGHTRED, William. The Circles of Proportion and the Horizontal Instrvment. London: Augustine Mathewes, 1633.

PEREIRA, Ana Carolina Costa. Aspectos Históricos da régua de cálculo para a construção de conceitos matemáticos. São Paulo: Livraria da Física, 2015. (História da Matemática para o Ensino).

QUEIROZ, Tereza Aline Pereira de. Aprender a saber na Idade Média. In: Trivium e quadrivium: as artes liberais na Idade Média. Cotia: Ibis, 1999, p. 11-31.

ROCHE, John J.. The radius astronomicus in England. Annals Of Science, [S.L.], v. 38, n. 1, p. 1-32, jan. 1981. Informa UK Limited. http://dx.doi.org/10.1080/00033798100200101. Disponível em: https://www.tandfonline.com/doi/abs/10.1080/00033798100200101. Acesso em: 20 out. 2020.

ROSS, Richard P.. The Social and Economic Causes of the Revolution in the Mathematical Sciences in Mid-Seventeenth-Century England. Journal Of British Studies, [S.L.], v. 15, n. 1, p. 46-66, 1975. Cambridge University Press (CUP). http://dx.doi.org/10.1086/385678.

ROSSI, Paolo. O nascimento da ciência moderna na Europa. Bauru: Edusc, 2001. Tradução de: Antonio Angonese. 
SAITO, Fumikazu; DIAS, Marisa da Silva. Interface entre História da Matemática e ensino: uma atividade desenvolvida com base num documento do século XVI. Ciência \& Educação, v.19, no1, p. 89-111, 2013.

SANTOS, Andressa Gomes dos; PEREIRA, Ana Carolina Costa. A INCORPORAÇÃO DA RÉGUA DE CÁLCULO NO ENSINO DE MULTIPLICAÇÃO ATRAVÉS DA SUA CONSTRUÇÃO E DO SEU MANUSEIO. Boletim Cearense de Educação e História da Matemática, [S.L.], v. 7, n. 20, p. 357-369, 12 jul. 2020. Boletim Cearense de Educacao e Historia da Matematica - BOCEHM. http://dx.doi.org/10.30938/bocehm.v7i20.2827. Disponível em: https://revistas.uece.br/index.php/BOCEHM/article/view/2827. Acesso em: 12 jul. 2020a.

SANTOS, Andressa Gomes dos; PEREIRA, Ana Carolina Costa. A construção da escala de Gunter com o auxílio do GeoGebra. In: XX ENCONTRO DE PÓSGRADUAÇÃO E PESQUISA, 20., 2020, Fortaleza. Anais [...] . Fortaleza: Unifor, 2020b. p. 1-6.

SILVA, Isabelle Coelho da. Um estudo da incorporação de textos originais para a educação matemática: buscando critérios na articulação entre história e ensino. 2018. 92 f. Dissertação (Mestrado) - Curso de Matemática, Instituto Federal do Ceará, Fortaleza, 2018. Disponível em: <http://pgecm.fortaleza.ifce.edu.br/wpcontent/uploads/2018/11/Disserta\%C3\%A7\%C3\%A3o-Isabelle-Coelho-da-Silva.pdf >. Acesso em: 19 maio 2019.

WARD, John. The lives of the professors of Gresham College: to which is prefixed the life of the founder, sir Thomas Gresham. London: John Moore, 1740.

Recebido em: 24 / 02 / 2021

Aprovado em: 14 / 04 / 2021 